ANL -6320

\title{
ANL -6320
}

\section{Argonne Rational Laboratorn}

THE DIFFUSION OF CHROMIUM

IN GAMMA URANIUM

by

S. J. Rothman 


\section{DISCLAIMER}

This report was prepared as an account of work sponsored by an agency of the United States Government. Neither the United States Government nor any agency Thereof, nor any of their employees, makes any warranty, express or implied, or assumes any legal liability or responsibility for the accuracy, completeness, or usefulness of any information, apparatus, product, or process disclosed, or represents that its use would not infringe privately owned rights. Reference herein to any specific commercial product, process, or service by trade name, trademark, manufacturer, or otherwise does not necessarily constitute or imply its endorsement, recommendation, or favoring by the United States Government or any agency thereof. The views and opinions of authors expressed herein do not necessarily state or reflect those of the United States Government or any agency thereof. 


\section{DISCLAIMER}

Portions of this document may be illegible in electronic image products. Images are produced from the best available original document. 


\section{LEGAL NOTICE}

This report was prepared as an account of Government sponsored work. Neither the United States, nor the Commission, nor any person acting on behalf of the Commission:

A. Makes any warranty or representation, expressed or implied, with respect to the accuracy, completeness, or usefulness of the information contained in this report, or that the use of any information, apparatus, method, or process disclosed in this report may not infringe privately owned rights; or

B. Assumes any liabilities with respect to the use of, or for damages resulting from the use of any information, apparatus, method, or process disclosed in this report.

As used in the above, "person acting on behalf of the Commission" includes any employee or contractor of the Commission, or employee of such contractor, to the extent that such employee or contractor of the Commission, or employee of such contractor prepares, disseminates, or provides access to, any information pursuant to his employment or contract with the Commission, or his employment with such contractor.

Price 50 Cents. Available from the office of Technical Services, Department of Commerce, Washington 25, D.C. 
ANL-6320

Metals, Ceramics, and Mate rials (TID-4500, 16th Ed.) AEC Research and Development Report

\section{ARGONNE NATIONAL LABORATORY \\ 9700 South Cass Avenue \\ Argonne, Illinois}

\section{THE DIFFUSION OF CHROMIUM IN GAMMA URANIUM}

by

S. J. Rothman

Metallurgy Division

Partial Report - Metallurgy Program 4.1.27

Portions of the material in this report have appeared in the following Metallurgy Division Progress Report:

$$
\text { ANL-6330 } \quad 1960
$$

June 1961

Operated by The University of Chicago under

Contract W-31-109-eng-38 
TABLE OF CONTENTS

Page

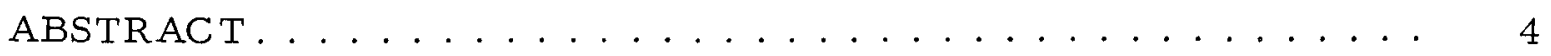

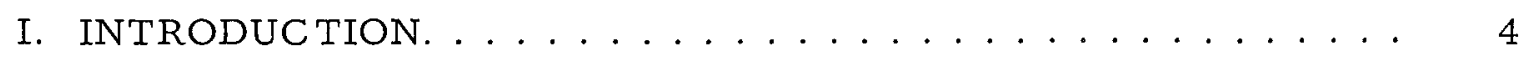

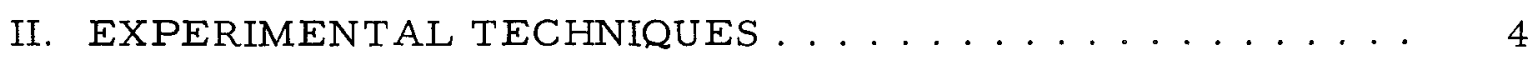

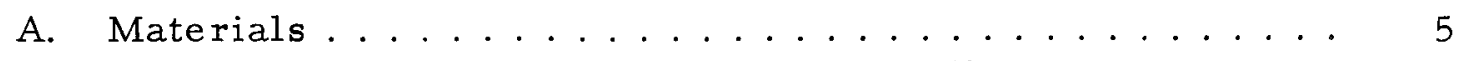

B. Preparation of Diffusion Couples and Diffusion

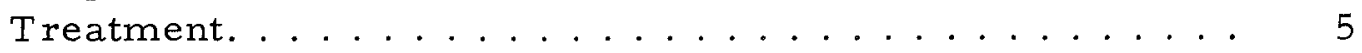

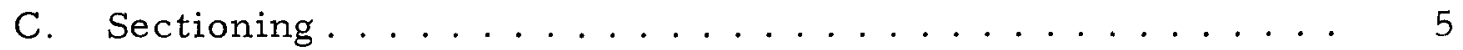

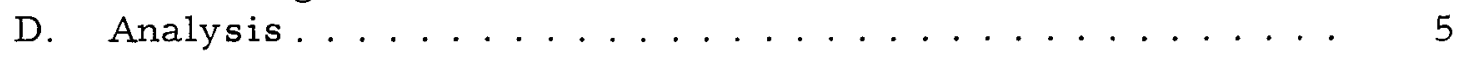

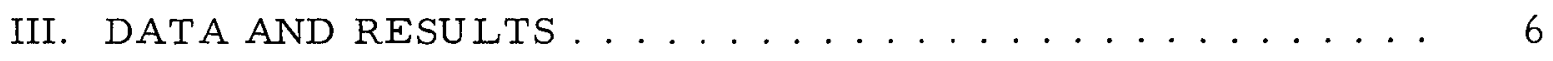

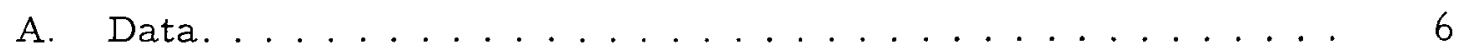

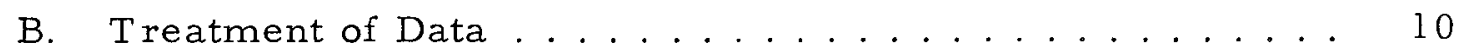

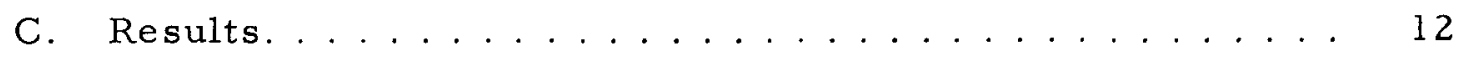

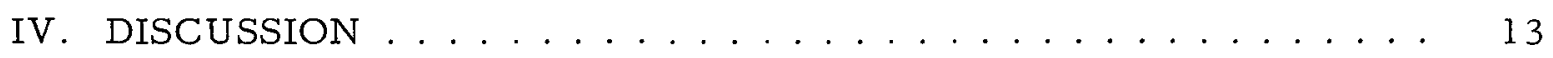

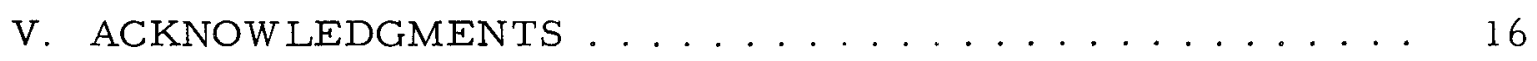

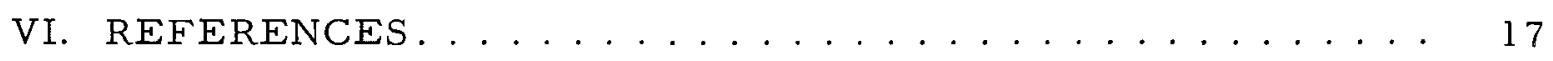




\section{LIST OF FIGURES}

No.

Title

Page

1. Typical Photopeak of $320-k e v \gamma$ ray from $\mathrm{Cr}^{51} \ldots \ldots$

2. Concentration-Penetration Plot for Samples 1 B and 2B.... 9

3. Concentration-Penetration Plot for Samples $3 \mathrm{~A}$ and 3 B ... . 9

4. Concentration-Penetration Plot for Samples 5A and 5B .... 10

5. Concentration-Penetration Plot for Sample 4B........ 11

6. Arrhenius Plot for Diffusion in Gamma and Beta Uranium . . 12

7. Schematic Sketch of Diffusion Sample Used by Mossé, Levy, and Adda .................... 15

\section{LIST OF TABLES}

No.

Title

Page

I. Penetration and Counting Data for Sample 1 B . . . . . . 7

II. Penetration and Counting Data for Sample 2B . . . . . . . 7

III. Penetration and Counting Data for Sample $3 \mathrm{~A} \ldots \ldots 7$

IV. Penetration and Counting Data for Sample 3B ....... 7

V. Penetration and Counting Data for Sample 4 B . . . . . . 8

VI. Penetration and Counting Data for Sample 5A ....... 8

VII. Penetration and Counting Data for Sample 5 B ....... 8

VIII. Diffusion Data ..................... 11 
THE DIFFUSION OF CHROMIUM IN GAMMA URANIUM

by

S. J. Rothman

\begin{abstract}
The diffusion of tracer amounts of chromium in gamma uranium has been studied by the radioactive tracersectioning method. The temperature dependence of the diffusion coefficient $D$ is given by

$$
\mathrm{D}=1.98 \times 10^{-3} \exp (-21,800 / \mathrm{RT}) \mathrm{cm}^{2} / \mathrm{sec} \text {, }
$$

in poor agreement with the data of Mossé, Lévy and Adda.(1) Possible reasons for the discrepancy are discussed, and it is concluded that the present data are to be preferred as a basis for theoretical studies of the diffusion of tracers in gamma uranium. The difference between the above activation energy and that for self-diffusion is also discussed; no conclusions are reached.
\end{abstract}

\title{
INTRODUCTION
}

This report is part of a program on the diffusion of various tracer elements in gamma uranium, $(2,3)$ which is intended to obtain fundamental data on the effect of solute valence and atomic size on solute diffusion parameters. Chromium was chosen as the diffusing isotope because, in addition to the fundamental aims of the study, it appeared worth while to check the existing data(1) on the diffusion of chromium in gamma uranium, since the reported diffusion coefficients (DC's) are very high, the plot of $\log \mathrm{D}$ vs $1 / \mathrm{T}$ is curved, and the method by which the DC's were obtained seems open to objections.

\section{EXPERIMENT AL TECHNIQUES}

The experimental techniques used were similar to those described before. $(2,3)$ Only the differences are mentioned. 
Materials

The uranium used was described in Reference 3. The tracer was electrolytic chromium, $99.2 \%$ pure (main impurity - about $0.4 \mathrm{w} / \mathrm{O} \mathrm{Fe}$ ), irradiated 16 days in a flux of $2 \times 10^{12} \mathrm{nv}$.

Preparation of Diffusion Couples and Diffusion Treatment

The diffusion couples were prepared, sealed off, and annealed as described in Reference 3 (see also References 2 and 4), except that for the evaporation of the tracer, the uranium samples were held face down above the tungsten spiral containing the chromium. Also, the samples were preheated for the diffusion anneal to a temperature slightly below the $\beta-\gamma$ transformation by hanging them in the furnace above the heat sink for about $10 \mathrm{~min}$; corrections for heating time were thus avoided.

$\underline{\text { Sectioning }}$

The sectioning was carried out as in References 2 and 3, except that more material was turned off the diameter and thicker sections were cut because the anticipated DC's were higher.

Analysis

The sections were analysed by counting the radioactive chromium isotope. They were first dissolved in $\mathrm{HCl}$, with $\mathrm{HNO}_{3}$ added to complete the dissolution. The solutions were made up to $2 \mathrm{ml}$, and $1-\mathrm{ml}$ aliquots were pipetted (with a micropipette) into flat Pyrex cups, $1 \frac{1}{16}$ in. in diameter and $\frac{3}{8}$ in. high. The cups were placed in a reproducible position about $\frac{5}{8}$ in. under the face of the scintillation crystal.

A scintillation counter, with a $1 \frac{1}{2}$-in.-diameter, 1-in.-thick $\mathrm{NaI}(\mathrm{T} 1)$ crystal, and a single channel analyzer was used to count the $320-\mathrm{kev}$ photopeak from $\mathrm{Cr}^{51}$. (5) The window of the analyzer could be opened wide enough to take in the entire peak with about $5 \mathrm{kev}$ to spare on either side. The position of the peak was plotted at the beginning of each day's counting, using a narrow window ( $5 \mathrm{kev}$ ); a typical peak is shown in Fig. 1. Plotting the peak after counting showed that gain changes were negligible. Also, the high voltage on the photomultiplier tube was adjusted to give the maximum count, and the linearity of the gain was checked regularly.

The first section of each sample (the second section on sample 1B; the first one was spilled) was counted before and after each succeeding section, (6) and the average of these two counts was used as a normalizing factor, to take care of changes in counter characteristics, decay, etc. 


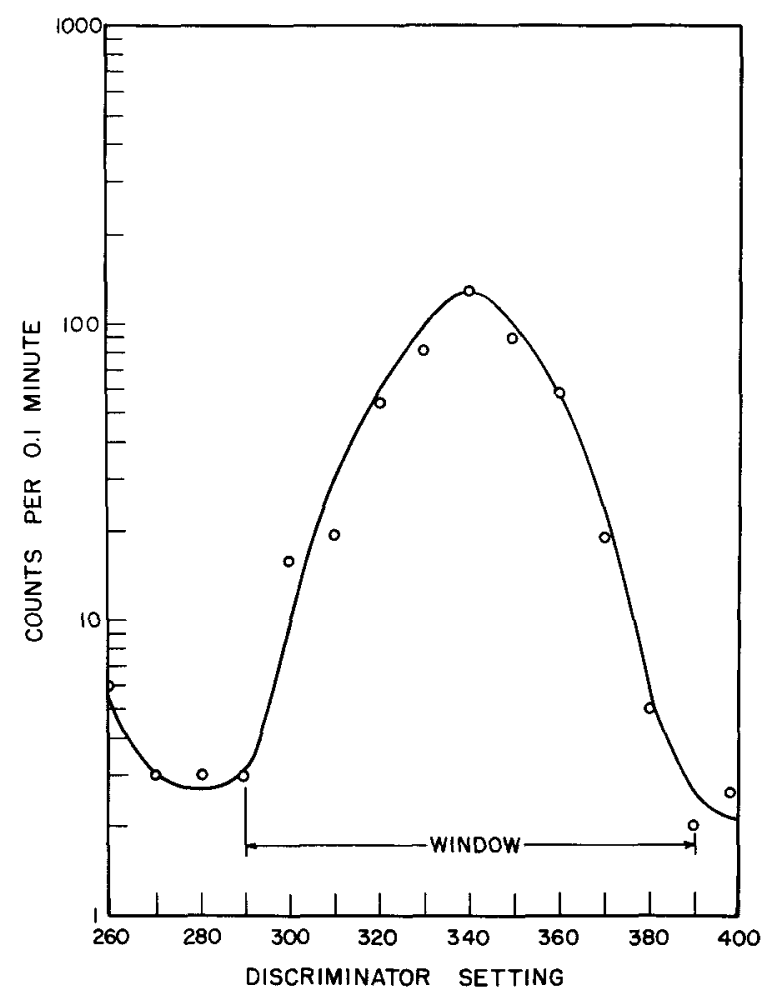

Fig. 1

Typical Photopeak of 320-kev $\gamma$ ray from $\mathrm{Cr}^{51}$.

30541

All counting rates were corrected for background. The activity in each section due to the uranium was determined by counting a pure solution of uranium, and was subtracted. A solution of $\mathrm{Cr}^{51}$ yielded

$3.12 \times 10^{5} \mathrm{cpm} / \mathrm{mg} \mathrm{Cr}$ under the same counting conditions, indicating that no section contained more than $0.1 \% \mathrm{Cr}$, and that the initial $\mathrm{Cr}$ layer was approximately 1.5 microns thick.

DATA AND RESULTS

Data

The data on section weights, counts per minute, etc., are given in Tables I to VII. The columns give:

1. section number,

2. weight of the section,

3. weight of the section corrected so that the sum of the weights of five sections equals the difference in the sample weight before and after cutting those sections,

4. the thickness of the section corrected for thermal expansion, so as to represent the section thickness at the temperature of the diffusion anneal (the corrections were made using the density data of Chiotti et al.), (7) 
Table I

PENETRATION AND COUNTING DATA FOR SAMPLE IB

\begin{tabular}{|c|c|c|c|c|c|c|c|c|c|c|c|c|}
\hline $\begin{array}{l}\text { Section } \\
\text { No }\end{array}$ & $\begin{array}{l}\text { Weight } \\
\text { (mg) }\end{array}$ & $\begin{array}{c}\text { Corrected } \\
\text { Weight } \\
\text { (mg) }\end{array}$ & $\begin{array}{l}\text { Thickness } \\
(\mathrm{cm})\end{array}$ & $X(\mathrm{~cm})$ & $x^{2}\left(\mathrm{~cm}^{2}\right)$ & $\begin{array}{l}\text { Total Counts } \\
\text { per Minutes } \\
\text { Counting Time }\end{array}$ & $\begin{array}{l}\text { Normalızıng } \\
\text { Factor }\end{array}$ & $\begin{array}{l}\text { Normalızed Total } \\
\text { Counts per } \\
\text { Minutes Countıng } \\
\text { Time }\end{array}$ & $\begin{array}{l}\text { Counts } \\
\text { per } \\
\text { Minute } \\
\text { (cpm) }\end{array}$ & $\begin{array}{l}\text { CPm Corrected } \\
\text { for Background }\end{array}$ & $\begin{array}{c}\mathrm{I} \\
\text { (cpm/mg) }\end{array}$ & $\begin{array}{c}\text { I } \\
\text { (Corrected } \\
\text { cpm/mg) }\end{array}$ \\
\hline $1^{*}$ & 481 & 492 & 002161 & 001080 & 00001166 & & & & & & & \\
\hline 2 & 567 & 580 & 002547 & 003435 & 00011799 & $15987 / 2$ & & $15697 / 2$ & 7843 & 7756 & 27358 & 27061 \\
\hline 3 & 577 & 590 & 002591 & 006004 & 00036048 & $12556 / 2$ & 15790 & $12482 / 2$ & 6241 & 6154 & 21331 & 21034 \\
\hline 4 & 560 & 573 & 002517 & 008557 & 00073222 & $12321 / 3$ & 15648 & $12360 / 3$ & 4180 & 4033 & 14404 & 14107 \\
\hline 5 & 546 & 558 & 002451 & 011042 & 00121926 & $12171 / 5$ & 15708 & $12162 / 5$ & 2432 & 2345 & 8590 & 8293 \\
\hline 6 & 533 & 541 & 002376 & 013455 & 00181037 & $10541 / 8$ & 15666 & $10566 / 8$ & 1321 & 1234 & 4630 & 4333 \\
\hline 7 & 558 & 566 & 002486 & 015886 & 00252365 & $7338 / 10$ & 15652 & $7359 / 10$ & 736 & 649 & 2326 & 2029 \\
\hline 8 & 583 & 592 & 002600 & 018429 & 00339628 & $3975 / 10$ & 15676 & $3980 / 10$ & 398 & 311 & 1067 & 770 \\
\hline 9 & 558 & 566 & 002486 & 020972 & 00439825 & $2424 / 10$ & 15694 & $2424 / 10$ & 242 & 155 & 556 & 259 \\
\hline $10^{*}$ & 575 & 583 & 002560 & 023496 & 00552062 & $1868 / 10$ & 15654 & $1873 / 10$ & 187 & 100 & 348 & 051 \\
\hline
\end{tabular}

Table II

PENETRATION AND COUNTING DATA FOR SAMPLE 2B

\begin{tabular}{|c|c|c|c|c|c|c|c|c|c|c|c|c|}
\hline $\begin{array}{l}\text { Section } \\
\text { No }\end{array}$ & $\begin{array}{l}\text { Weight } \\
\text { (mg) }\end{array}$ & $\begin{array}{l}\text { Corrected } \\
\text { Weight } \\
\text { (mg) }\end{array}$ & $\begin{array}{l}\text { Thickness } \\
(\mathrm{cm})\end{array}$ & $x(\mathrm{~cm})$ & $x^{2}\left(\mathrm{~cm}^{2}\right)$ & $\begin{array}{l}\text { Total Counts } \\
\text { per Minutes } \\
\text { CountIng TIme }\end{array}$ & $\begin{array}{l}\text { Normalızıng } \\
\text { Factor }\end{array}$ & $\begin{array}{l}\text { Normalızed Total } \\
\text { Counts per } \\
\text { Minutes Counting } \\
\text { Time }\end{array}$ & $\begin{array}{l}\text { Counts } \\
\text { per } \\
\text { Minute } \\
\text { (cpm) }\end{array}$ & $\begin{array}{l}\text { cpm Corrected } \\
\text { for Background }\end{array}$ & $\begin{array}{c}I \\
(\mathrm{cpm} / \mathrm{mg})\end{array}$ & $\begin{array}{c}\text { I } \\
\text { (Corrected } \\
\mathrm{cpm} / \mathrm{mg} \text { ) }\end{array}$ \\
\hline 1 & 493 & 502 & 00217 & 00108 & 0000117 & $11911 / 2$ & & $11814 / 2$ & 5907 & 5817 & 23598 & 23308 \\
\hline 2 & 562 & 572 & 00248 & 00341 & 000116 & $11875 / 2$ & 11926 & $11757 / 2$ & 5878 & 5788 & 20598 & 20308 \\
\hline 3 & 422 & 531 & 00230 & 00580 & 000336 & $13010 / 3$ & 11810 & $13014 / 3$ & 4338 & 4248 & 16276 & 15986 \\
\hline 4 & 539 & 548 & 00237 & 00813 & 000661 & $12291 / 4$ & 11704 & $12406 / 4$ & 3102 & 3012 & 11176 & 10886 \\
\hline 5 & 550 & 560 & 00242 & 01053 & 001109 & $10951 / 6$ & 11744 & $11016 / 6$ & 1836 & 1746 & 6349 & 6059 \\
\hline 6 & 556 & 564 & 00244 & 01296 & 001680 & $10783 / 10$ & 11798 & $10798 / 10$ & 1080 & 1000 & 3597 & 3307 \\
\hline 7 & 553 & 561 & 00243 & 01540 & 002372 & $9242 / 16$ & 11817 & $9240 / 16$ & 578 & 488 & 1765 & 1475 \\
\hline 8 & 577 & 585 & 00253 & 01787 & 003193 & $4975 / 15$ & 11820 & $4972 / 15$ & 331 & 241 & 835 & 545 \\
\hline $9^{*}$ & 560 & 568 & 00246 & 02037 & 004149 & $3493 / 15$ & 11730 & $3518 / 15$ & 234 & 144 & 514 & 224 \\
\hline $10^{*}$ & 572 & 580 & 00251 & 02286 & 005226 & $2790 / 15$ & 11824 & $2788 / 15$ & 186 & 96 & 336 & 046 \\
\hline
\end{tabular}

Table III

PENETRATION AND COUNTING DATA FOR SAMPLE 3A

\begin{tabular}{|c|c|c|c|c|c|c|c|c|c|c|c|c|}
\hline $\begin{array}{l}\text { Section } \\
\text { No }\end{array}$ & $\begin{array}{l}\text { Welght } \\
\text { (mg) }\end{array}$ & $\begin{array}{c}\text { Corrected } \\
\text { Weight } \\
\text { (mg) }\end{array}$ & $\begin{array}{l}\text { Thickness } \\
(\mathrm{cm})\end{array}$ & $X(\mathrm{~cm})$ & $x^{2}\left(\mathrm{~cm}^{2}\right)$ & $\begin{array}{l}\text { Total Counts } \\
\text { per Minutes } \\
\text { Counting Time }\end{array}$ & $\begin{array}{l}\text { Normalızıng } \\
\text { Factor }\end{array}$ & $\begin{array}{l}\text { Normalızed Total } \\
\text { Counts per } \\
\text { Mınutes Countıng } \\
\text { Tıme }\end{array}$ & $\begin{array}{l}\text { Counts } \\
\text { per } \\
\text { Minute } \\
\text { (cpm) }\end{array}$ & $\begin{array}{l}\text { cpm Corrected } \\
\text { for Background }\end{array}$ & $\underset{(\mathrm{cpm} / \mathrm{mg})}{\mathrm{I}}$ & $\begin{array}{c}I \\
\text { (Corrected } \\
\text { cpm/mg) }\end{array}$ \\
\hline 1 & 659 & 670 & 000992 & 000496 & 00000246 & $16736 / 2$ & & $16691 / 2$ & 8346 & 8252 & 25044 & 24749 \\
\hline 2 & 832 & 847 & 001254 & 001619 & 00002621 & 10037 & 16778 & 9985 & 9985 & 9891 & 23776 & 23481 \\
\hline 3 & 869 & 884 & 001309 & 002900 & 00008410 & $16310 / 2$ & 16720 & $16282 / 2$ & 8141 & 8047 & 18520 & 18225 \\
\hline 4 & 858 & 873 & 001293 & 004201 & 00017648 & $11977 / 2$ & 16664 & $11996 / 2$ & 5998 & 5904 & 13762 & 13467 \\
\hline 5 & 854 & 869 & 001287 & 005492 & 00030162 & $11296 / 3$ & 16648 & $11325 / 3$ & 3775 & 3681 & 8621 & 8326 \\
\hline 6 & 816 & 831 & 001231 & 006751 & 00045576 & $10672 / 5$ & 16650 & $10698 / 5$ & 2140 & 2046 & 5015 & 4720 \\
\hline 7 & 856 & 872 & 001291 & 008012 & 00064192 & $10118 / 8$ & 16772 & $10069 / 8$ & 1259 & 1165 & 2722 & 2427 \\
\hline 8 & 898 & 915 & 001355 & 009336 & 00087161 & $6952 / 10$ & 16725 & $6938 / 10$ & 694 & 600 & 1336 & 1041 \\
\hline $9^{*}$ & 885 & 901 & 001334 & 010680 & 0011406 & $3740 / 10$ & 16670 & $3745 / 10$ & 374 & 280 & 633 & 338 \\
\hline $10^{*}$ & 843 & 859 & 001271 & 011983 & 0014359 & $2550 / 10$ & 16638 & $2558 / 10$ & 256 & 162 & 380 & 085 \\
\hline
\end{tabular}

Table IV

PENETRATION AND COUNTING DATA FOR SAMPLE 3B

\begin{tabular}{|c|c|c|c|c|c|c|c|c|c|c|c|c|}
\hline $\begin{array}{l}\text { Section } \\
\text { No }\end{array}$ & $\begin{array}{l}\text { Welght } \\
\text { (mg) }\end{array}$ & $\begin{array}{c}\text { Corrected } \\
\text { Weight } \\
\text { (mg) }\end{array}$ & $\begin{array}{c}\text { Thickness } \\
(\mathrm{cm})\end{array}$ & $X(\mathrm{~cm})$ & $x^{2}\left(\mathrm{~cm}^{2}\right)$ & $\begin{array}{l}\text { Total Counts } \\
\text { per Minutes } \\
\text { Countıng TIme }\end{array}$ & $\begin{array}{l}\text { Normalızıng } \\
\text { Factor }\end{array}$ & $\begin{array}{l}\text { Normalızed Total } \\
\text { Counts per } \\
\text { Mınutes Countıng } \\
\text { TIme }\end{array}$ & $\begin{array}{l}\text { Counts } \\
\text { per } \\
\text { Minute } \\
(\mathrm{cpm})\end{array}$ & $\begin{array}{l}\mathrm{cpm} \text { Corrected } \\
\text { for Background }\end{array}$ & $\underset{\text { (cpm/mg) }}{\mathrm{I}}$ & $\begin{array}{c}\text { I } \\
\text { (Corrected } \\
\mathrm{cpm} / \mathrm{mg} \text { ) }\end{array}$ \\
\hline 1 & 890 & 901 & 001299 & 000650 & 00000422 & 12711 & & 12564 & 12564 & 12476 & 28036 & 27867 \\
\hline $2^{*}$ & 740 & 748 & 001078 & 001838 & 00003378 & 10460 & 12659 & 10381 & 10381 & 10293 & 27819 & 27650 \\
\hline 3 & 848 & 858 & 001237 & 002995 & 00008970 & $17500 / 2$ & 12594 & $17458 / 2$ & 8729 & 8641 & 20380 & 20211 \\
\hline 4 & 859 & 869 & 001253 & 004241 & 00017986 & $12785 / 2$ & 12674 & $12674 / 2$ & 6337 & 6249 & 14549 & 14380 \\
\hline 5 & 883 & 894 & 001289 & 005512 & 00030382 & $12845 / 3$ & 12616 & $12792 / 3$ & 4264 & 4176 & 9459 & 9290 \\
\hline 6 & 980 & 999 & 001440 & 006876 & 00047279 & $10833 / 4$ & 12488 & $10899 / 4$ & 2725 & 2637 & 5382 & 5213 \\
\hline 7 & 916 & 934 & 001346 & 008269 & 00068376 & $11245 / 9$ & 12558 & $11250 / 9$ & 1250 & 1162 & 2537 & 2368 \\
\hline 8 & 897 & 915 & 001319 & 009602 & 00092198 & $10122 / 16$ & 12576 & $10112 / 16$ & 632 & 544 & 1213 & 1044 \\
\hline$g^{*}$ & 900 & 918 & 001323 & 010923 & 00119312 & $4062 / 11$ & 12472 & $4092 / 11$ & 372 & 284 & 631 & 462 \\
\hline $10^{\text {* }}$ & 858 & 875 & 001261 & 012214 & 00149182 & $2385 / 10$ & 12425 & $2412 / 10$ & 241 & 153 & 357 & 188 \\
\hline
\end{tabular}

* Not used in calculating D 
Table $\mathbf{Y}$

PENETRATION AND COUNTING DATA FOR SAMPLE 4B

\begin{tabular}{|c|c|c|c|c|c|c|c|c|c|c|c|c|}
\hline $\begin{array}{l}\text { Section } \\
\text { No }\end{array}$ & $\begin{array}{l}\text { Weight } \\
\text { (mg) }\end{array}$ & $\begin{array}{c}\text { Corrected } \\
\text { Welght } \\
\text { (mg) }\end{array}$ & $\begin{array}{l}\text { Thickness } \\
(\mathrm{cm})\end{array}$ & $X(\mathrm{~cm})$ & $x^{2}\left(\mathrm{~cm}^{2}\right)$ & $\begin{array}{c}\text { Jotal Counts } \\
\text { per Minutes } \\
\text { Countıng Tıme }\end{array}$ & $\begin{array}{l}\text { Normalizıng } \\
\text { Factor }\end{array}$ & $\begin{array}{l}\text { Normalized Total } \\
\text { Counts per } \\
\text { Minutes Counting } \\
\text { Time }\end{array}$ & $\begin{array}{l}\text { Counts } \\
\text { per } \\
\text { Minute } \\
\text { (cpm) }\end{array}$ & $\begin{array}{l}\text { cpm Corrected } \\
\text { for Background }\end{array}$ & $\frac{I}{\text { (cpm/mg) }}$ & $\begin{array}{c}\text { I } \\
\text { (Corrected } \\
\text { cpm/mg) }\end{array}$ \\
\hline 1 & 427 & 431 & 0008174 & 0004087 & 000001670 & $12292 / 2$ & & $12159 / 2$ & 6080 & 5988 & 28047 & 27856 \\
\hline 2 & 641 & 647 & 0012270 & 0014309 & 000020475 & $17333 / 2$ & 12324 & $1710 \mathrm{l} / 2$ & 8550 & 8458 & 26390 & 26199 \\
\hline 3 & 720 & 727 & 0013788 & 0027338 & 000074737 & $17336 / 2$ & 12199 & $17279 / 2$ & 8640 & 8548 & 23744 & 23553 \\
\hline 4 & 672 & 679 & 0012877 & 0040670 & 000165405 & $13239 / 2$ & 12048 & $13361 / 2$ & 6680 & 6588 & 19607 & 19416 \\
\hline 5 & 733 & 740 & 0014034 & 0054126 & 000292962 & $11334 / 2$ & 12069 & $11418 / 2$ & 5709 & 5617 & 15326 & 15135 \\
\hline 6 & 467 & 480 & 0009103 & 0065695 & 000431583 & $10427 / 4$ & 12101 & $10477 / 4$ & 2619 & 2527 & 10822 & 10631 \\
\hline 7 & 661 & 679 & 0012877 & 0076684 & 000588044 & $11021 / 4$ & 12142 & $11036 / 4$ & 2759 & 2667 & 8070 & 7879 \\
\hline 8 & 694 & 713 & 0013522 & 0089844 & 000807913 & $11507 / 6$ & 12165 & $11501 / 6$ & 1917 & 1825 & 5259 & 5068 \\
\hline 9 & 678 & 697 & 0013219 & 0103255 & 001066160 & $11646 / 10$ & & $11646 / 10$ & 11646 & 1073 & 3165 & 2974 \\
\hline 10 & 705 & 725 & 0013750 & 0116739 & 001362799 & $7556 / 10$ & & $7556 / 10$ & 7556 & 664 & 1884 & 1693 \\
\hline
\end{tabular}

Table VI

PENETRATION AND COUNTING DATA FOR SAMPLE 5 A

\begin{tabular}{|c|c|c|c|c|c|c|c|c|c|c|c|c|}
\hline $\begin{array}{l}\text { Section } \\
\text { No }\end{array}$ & $\begin{array}{l}\text { Weight } \\
\text { (mg) }\end{array}$ & $\begin{array}{l}\text { Corrected } \\
\text { Weight } \\
\text { (mg) }\end{array}$ & $\begin{array}{c}\text { Thickness } \\
\text { (cm) }\end{array}$ & $X(\mathrm{~cm})$ & $x^{2}\left(\mathrm{~cm}^{2}\right)$ & $\begin{array}{l}\text { Total Counts } \\
\text { per Minutes } \\
\text { Countıng Time }\end{array}$ & $\begin{array}{l}\text { Normalızıng } \\
\text { Factor }\end{array}$ & $\begin{array}{l}\text { Normalızed Total } \\
\text { Counts per } \\
\text { Mınutes Countıng } \\
\text { Tıme }\end{array}$ & $\begin{array}{l}\text { Counts } \\
\text { per } \\
\text { Minute } \\
\text { (cpm) }\end{array}$ & $\begin{array}{l}\text { cpm Corrected } \\
\text { for Background }\end{array}$ & $\underset{(\mathrm{cpm} / \mathrm{mg})}{\mathrm{I}}$ & $\begin{array}{c}\text { I } \\
\text { (Corrected } \\
\text { cpm/mg) }\end{array}$ \\
\hline 1 & 523 & 532 & 001480 & 000740 & 00000548 & $12774 / 2$ & & $12661 / 2$ & 6330 & 6242 & 23870 & 23680 \\
\hline 2 & 655 & 665 & 001850 & 002405 & 00005784 & $13300 / 2$ & 12792 & $13164 / 2$ & 6582 & 6494 & 19829 & 1919 \\
\hline 3 & 628 & 638 & 001775 & 004218 & 00017791 & $14090 / 3$ & 12756 & $13985 / 3$ & 4662 & 4574 & 14567 & 14357 \\
\hline 4 & 656 & 667 & 001856 & 006033 & 00036397 & $11738 / 4$ & 12588 & $11805 / 4$ & 2951 & 2863 & 8729 & 8519 \\
\hline 5 & 639 & 649 & 001806 & 007864 & 00061842 & $10388 / 7$ & 12567 & $10466 / 7$ & 1495 & 1407 & 4404 & 4194 \\
\hline 6 & 521 & 526 & 001463 & 009499 & 00090231 & $5989 / 10$ & 12586 & $6025 / 10$ & 602 & 514 & 1973 & 1763 \\
\hline 7 & 645 & 652 & 001814 & 011137 & 00124033 & $3824 / 10$ & 12544 & $3860 / 10$ & 386 & 298 & 924 & 714 \\
\hline $8^{\alpha}$ & 628 & 634 & 001764 & 012926 & 00167081 & $2254 / 10$ & 12640 & $2258 / 10$ & 226 & 138 & 439 & 229 \\
\hline $9^{*}$ & 655 & 668 & 001858 & 014737 & 00217179 & $1968 / 11$ & 12710 & $1960 / 11$ & 178 & 90 & 275 & 065 \\
\hline $10^{*}$ & 678 & 685 & 001906 & 016619 & 00276191 & & & & & & & \\
\hline
\end{tabular}

${ }^{\circ}$ Not used in calculatıng D

Table VII

PENETRATION AND COUNTING DATA FOR SAMPLE $5 B$

\begin{tabular}{|c|c|c|c|c|c|c|c|c|c|c|c|c|}
\hline $\begin{array}{l}\text { Section } \\
\text { No }\end{array}$ & $\begin{array}{l}\text { Werght } \\
\text { (mg) }\end{array}$ & $\begin{array}{c}\text { Corrected } \\
\text { Weight } \\
\text { (mg) }\end{array}$ & $\begin{array}{c}\text { Thickness } \\
(\mathrm{cm})\end{array}$ & $X(\mathrm{~cm})$ & $x^{2}\left(\mathrm{~cm}^{2}\right)$ & $\begin{array}{c}\text { Total Counts } \\
\text { per Minutes } \\
\text { Counting Time }\end{array}$ & $\begin{array}{l}\text { Normalızıng } \\
\text { Factor }\end{array}$ & $\begin{array}{l}\text { Normalızed Total } \\
\text { Counts per } \\
\text { Minutes Countıng } \\
\text { TIme }\end{array}$ & $\begin{array}{l}\text { Counts } \\
\text { per } \\
\text { Minute } \\
\text { (cpm) }\end{array}$ & $\begin{array}{l}\text { cpm Corrected } \\
\text { for Background }\end{array}$ & $\underset{\text { (cpm/mg) }}{I}$ & $\begin{array}{c}\text { I } \\
\text { (Corrected } \\
\text { cpm/mg) }\end{array}$ \\
\hline 1 & 556 & 563 & 001558 & 000779 & 00000607 & $12142 / 2$ & & $12184 / 2$ & 6092 & 5996 & 21568 & 21255 \\
\hline 2 & 648 & 657 & 001813 & 002464 & 00006071 & $12130 / 2$ & 12238 & $12076 / 2$ & 6038 & 5942 & 18340 & 18027 \\
\hline 3 & 658 & 667 & 001846 & 004294 & 00018438 & $13099 / 3$ & 12262 & $13016 / 3$ & 4337 & 4241 & 12891 & 12578 \\
\hline 4 & 644 & 653 & 001808 & 006121 & 00037467 & $12641 / 5$ & 12154 & $12672 / 5$ & 2534 & 2438 & 7571 & 7258 \\
\hline 5 & 664 & 673 & 001863 & 007957 & 00063314 & $11260 / 8$ & 12126 & $11314 / 8$ & 1414 & 1318 & 3970 & 3657 \\
\hline 6 & 553 & 567 & 001569 & 009672 & 00093548 & $5967 / 10$ & 12166 & $5976 / 10$ & 598 & 502 & 1816 & 1503 \\
\hline 7 & 649 & 666 & 001843 & 011379 & 00129481 & $3659 / 10$ & 12176 & $3661 / 10$ & 366 & 270 & 832 & 519 \\
\hline $8^{*}$ & 637 & 654 & 001810 & 013205 & 00174372 & $2330 / 10$ & 12150 & $2336 / 10$ & 234 & 138 & 433 & 120 \\
\hline $9^{*}$ & 640 & 657 & 001818 & 015019 & 00225570 & $1911 / 10$ & 12178 & $1912 / 10$ & 191 & 95 & 297 & -016 \\
\hline $10^{*}$ & 634 & 651 & 001802 & 016829 & 00283215 & $887 / 5$ & 12216 & 88515 & 177 & 81 & 256 & 057 \\
\hline
\end{tabular}

"Not used in calculating D 
5. the distance of the center of the section from the original interface $(X)$,

6. the square of the above,

7. total counts and counting time in minutes,

8. normalizing factor (vide supra),

9. normalized total counts and counting time in minutes,

10. counts per minute (cpm),

11. cpm corrected for background,

12. the specific activity of the section, $\mathrm{I}$, in $\mathrm{cpm} / \mathrm{millig} \mathrm{ram}$ (cpm/mg),

13. I, corrected for U activity.

The data of these tables are shown in the customary log I vs $X^{2}$ plots in Figs. 2-5.

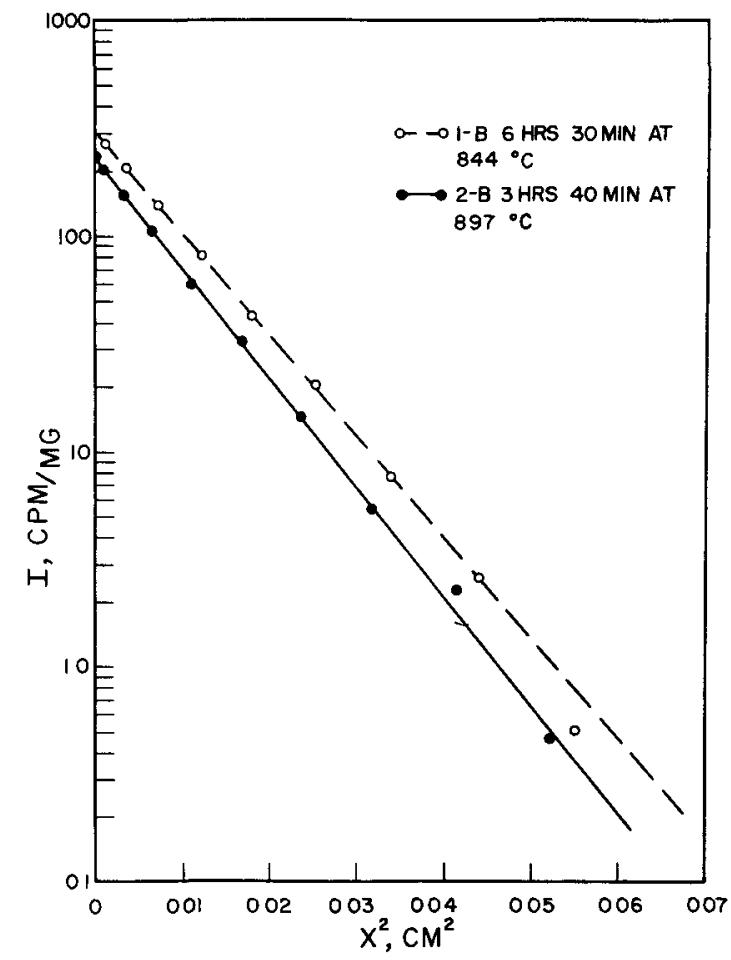

30147

Fig. 2 Concentration-Penetration Plot for Samples $1 \mathrm{~B}$ and $2 \mathrm{~B}$.

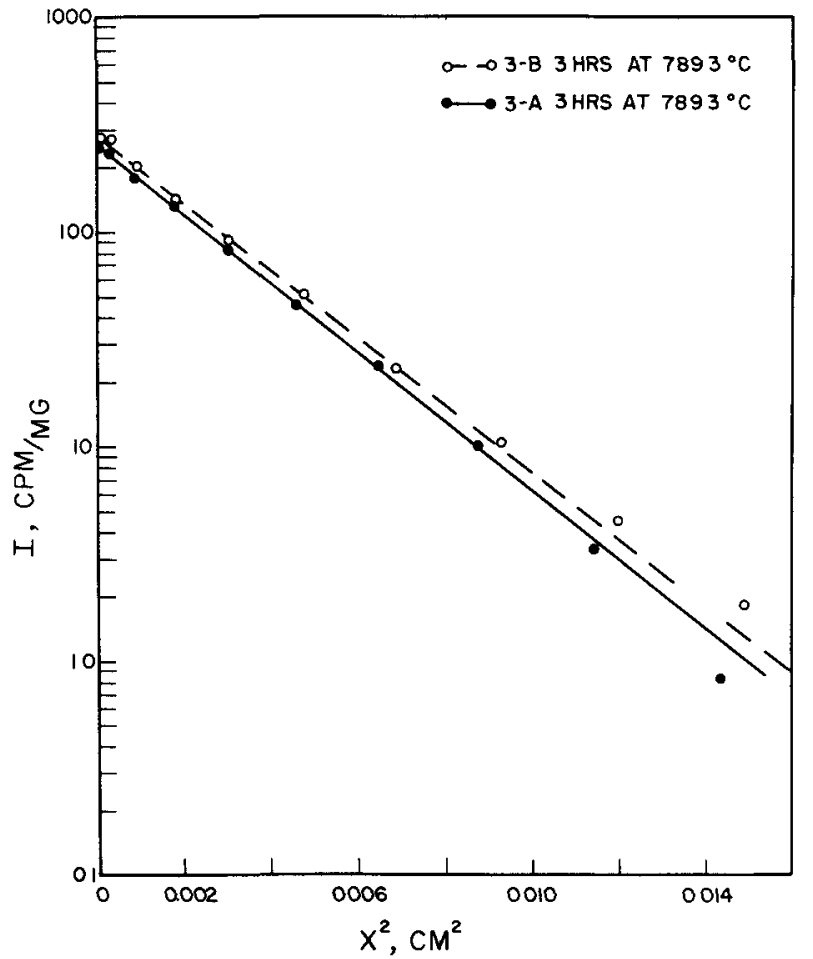

30146

Fig. 3 Concentration-Penetration Plot for Samples $3 \mathrm{~A}$ and $3 \mathrm{~B}$. 


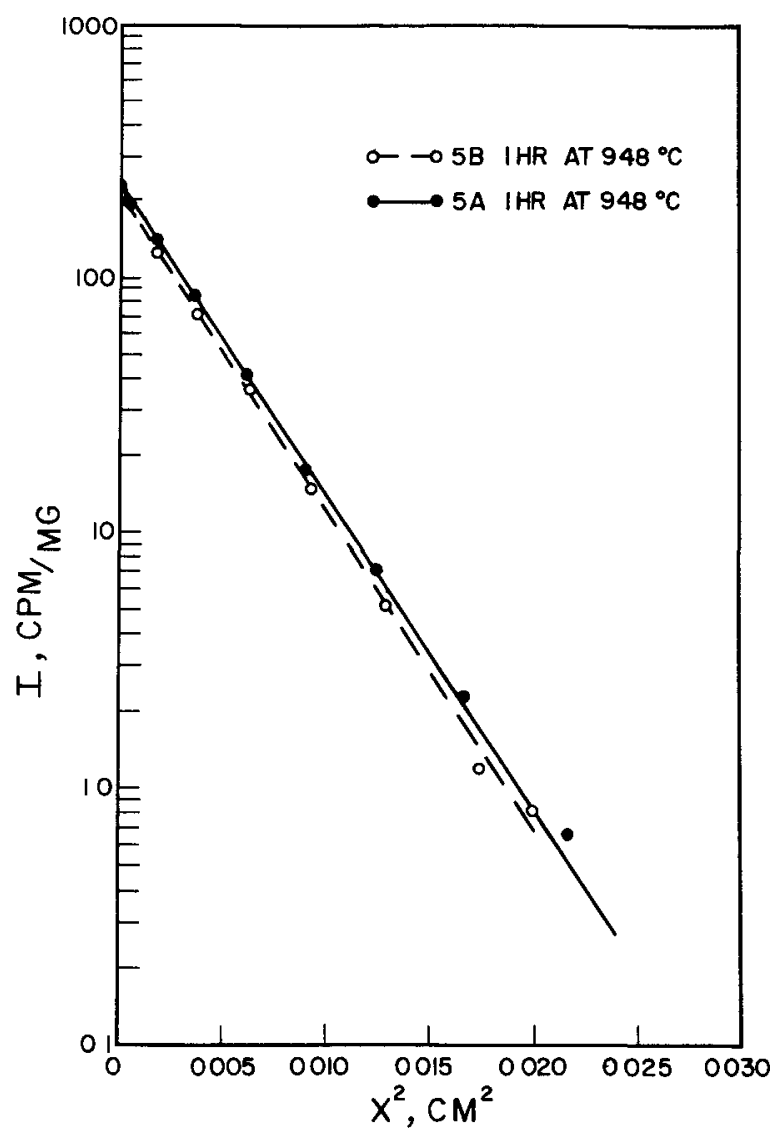

Fig. 4

Concentration-Penetration Plot for Samples $5 \mathrm{~A}$ and $5 \mathrm{~B}$.

30148

The annealing temperatures, annealing times, specimen diameters, DC's, and micrometer corrections are given in Table VIII. The times given were not corrected for warming up, except for samples $5 \mathrm{~A}$ and $5 \mathrm{~B}$, where formulae similar to Armstrong's(8) were used. The temperatures are accurate to $\pm 1{ }^{\circ} \mathrm{C}$.

Treatment of Data

Diffusion coefficients were calculated by the method of least squares from the equation(3)

$$
\log _{10} I=\log _{10} k^{\prime}-X^{2} / 9.212 D t
$$

where $k^{\prime}$ is a constant for any one sample, $D$ is the diffusion coefficient, and the annealing time. The DC's were corrected so that the total thickness removed equalled the thickness removed as measured by the mic rometer. (2) 


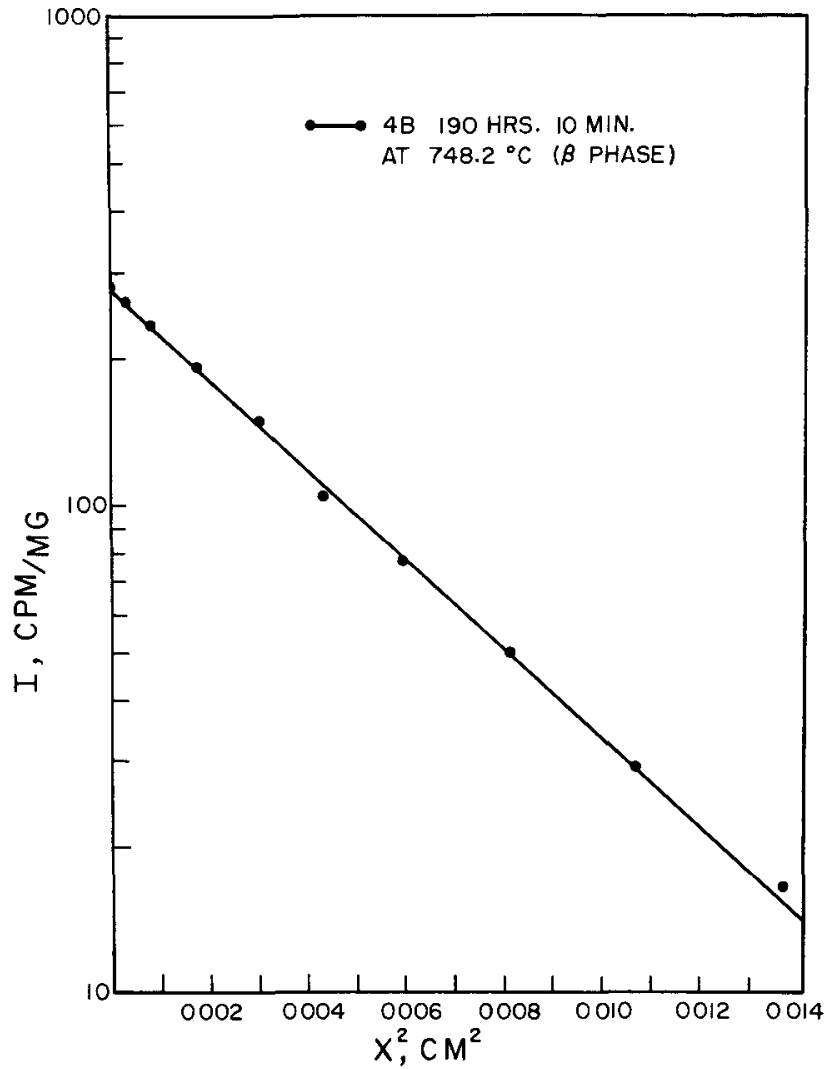

Fig. 5

Concentration-Penetration Plot for Sample $4 \mathrm{~B}$.

30185

Table VIII

DIFFUSION DATA

\begin{tabular}{|c|c|c|c|c|c|}
\hline $\begin{array}{c}\text { Sample } \\
\text { No. }\end{array}$ & $\begin{array}{c}\text { Annealing } \\
\text { Temp }\left({ }^{\circ} \mathrm{C}\right)\end{array}$ & $\begin{array}{c}\text { Annealing } \\
\text { Time } \\
(\mathrm{sec})\end{array}$ & $\begin{array}{c}\text { Diameter } \\
(\mathrm{cm})\end{array}$ & $\begin{array}{c}\mathrm{DC} \\
\left(\mathrm{cm}^{2} / \mathrm{sec}\right)\end{array}$ & $\begin{array}{c}\text { Corrected* } \\
\text { DC }\left(\mathrm{cm}^{2} / \mathrm{sec}\right)\end{array}$ \\
\hline lB & 844 & $2.34 \times 10^{4}$ & 0.403 & $0.982 \times 10^{-7}$ & $1.04 \times 10^{-7}$ \\
2B & 897 & $1.32 \times 10^{4}$ & 0.407 & $1.61 \times 10^{-7}$ & $1.60 \times 10^{-7}$ \\
3A & 789.3 & $1.08 \times 10^{4}$ & 0.692 & $6.33 \times 10^{-8}$ & $6.39 \times 10^{-8}$ \\
3B & 789.3 & $1.08 \times 10^{4}$ & 0.702 & $6.48 \times 10^{-8}$ & $6.77 \times 10^{-8}$ \\
4B & 748.2 & $6.846 \times 10^{5}$ & 0.609 & $1.77 \times 10^{-9}$ & $1.78 \times 10^{-9}$ \\
5A & 948 & $3.60 \times 10^{3}$ & 0.508 & $2.45 \times 10^{-7}$ & $2.52 \times 10^{-7}$ \\
5B & 948 & $3.60 \times 10^{3}$ & 0.509 & $2.43 \times 10^{-7}$ & $2.55 \times 10^{-7}$ \\
\hline
\end{tabular}

$* D$ corrected $=D\left(\frac{\text { Total thickness removed according to micrometer }}{\text { Total thickness removed according to weight }}\right)^{2}$

The last one, two, or three points deviated from the straight line on the penetration plots. Such points, as well as point 2 of sample $3 B$, were not used in the calculation of $\mathrm{D}$. Samples $1 \mathrm{~A}$ and $2 \mathrm{~A}$ were discarded because the sections cut from them were too thin. 
$\underline{\text { Results }}$

The DC's are tabulated in Table VIII and plotted vs $1 / \mathrm{T}$ in Fig. 6 , together with other data on diffusion in uranium. Fitting the results to an Arrhenius type equation:

$$
D=D_{0} \exp (-Q / R T)
$$

one obtains

$$
\begin{aligned}
& \mathrm{D}_{0}=1.98_{-0.53}^{+0.79} \times 10^{-3} \mathrm{~cm}^{2} / \mathrm{sec} \\
& Q=21,800 \pm 760 \mathrm{cal} / \mathrm{mol}
\end{aligned}
$$

The above errors are standard, not probable, errors. The total errors in the individual DC's are estimated to be: counting - $1 \%$, annealing time $-2 \%$, temperature - $1 \%$, weighing $-2 \%$, alignment $-1 \%$, total $-3 \frac{1}{2} \%$.

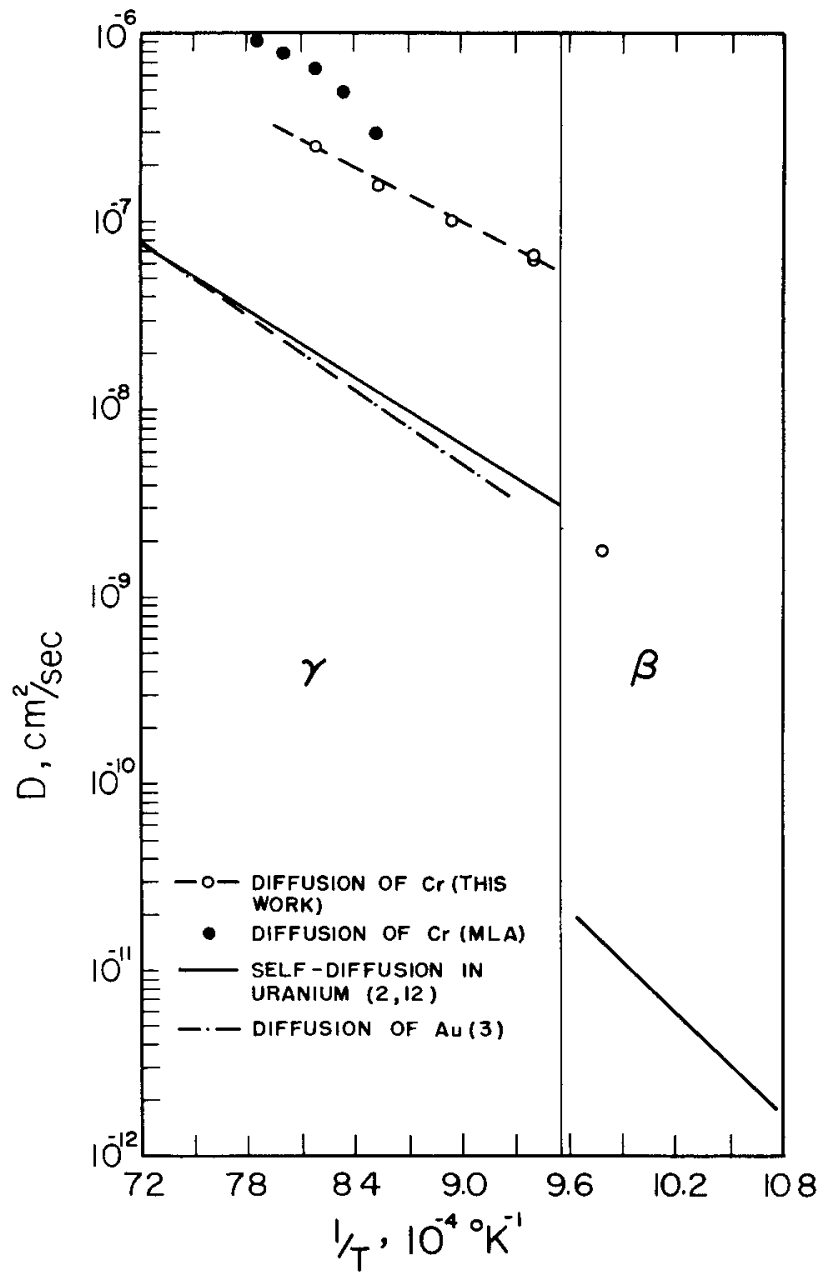

Fig. 6

Arrhenius Plot for Diffusion in Gamma and Beta Uranium 


\section{DISCUSSION}

The penetration plots (Figs. 2-5) are good straight lines, indicating that grain-boundary diffusion is negligible. The diffusion of chromium in beta uranium takes place by isotropic volume diffusion, although selfdiffusion in beta uranium displays both a grain-boundary component and anisotropy. There is a break in the $\log D(\mathrm{Cr})-1 / \mathrm{T}$ curve at the $\gamma-\beta$ trans formation, although the diffusion of chromium in beta uranium is about one hundred times as fast as self-diffusion (Fig. 6).

The activation energy for the diffusion of $\mathrm{Cr}$ is less by $4.7 \mathrm{kcal} / \mathrm{mol}$ (or $20 \%$ ) than that for self-diffusion. The opposite is expected from correlations of activation energies with solute-solvent valence differences, (9) since the valence of chromium is estimated to be less than that of uranium, and the correlation (observed on the diffusion of substitutional impurities in silver) shows that

$$
Q_{\text {solute }}<Q_{\text {solvent }}
$$

if

$$
\text { valence }_{\text {solute }}>\text { valence } \text { solvent } \quad \text {. }
$$

Correlation of the activation energy for solute diffusion with the atomic radius of the solute [for substitutional impurities diffusing in copper(10)] shows that

$$
Q_{\text {solute }}<Q_{\text {solvent }}
$$

if

$$
r_{\text {solute }}>r_{\text {solvent }}
$$

in the present case,

$$
Q_{\mathrm{Cr}}<Q_{\text {self }} \quad,
$$

although

$$
\mathrm{r}_{\mathrm{Cr}}<\mathrm{r}_{\mathrm{U}}
$$

Diffusion by an interstitial mechanism could explain the present results, including the fast pure volume diffusion in the beta phase; unfortunately, the interstices in both beta and gamma uranium are too small to hold a chromium atom. 
The ring mechanism, proposed as a mechanism for self-diffusion in gamma uranium (2) and in chromium, (11) provides a pleasantly simpleminded explanation for these data. In this mechanism, the activation energy is used to "squeeze" the atoms of the ring by their stationary neighbors and to push these neighbors apart. This should take less energy for chromium, since the chromium atom is smaller. The values of $D_{0}$ for selfdiffusion and the diffusion of chromium are nearly the same because the most influential term in $\mathrm{D}_{0}$, the "entropy of ring synchronization, "(11) should not change much with the atom involved. This hypothesis, like all ring-diffusion talk, is highly tenuous and tentative.

The present data do not agree with those of Mossé, Lévy, and Adda(I) who found $D_{0}=0.7 \mathrm{~cm}^{2} / \mathrm{sec}, Q=34,000 \mathrm{cal} / \mathrm{mol}$, and much higher DC's. The discrepancy can be analyzed as follows.

First, the two works measure different things. The present work concerns the diffusion of chromium in gamma uranium at very small concentrations and negligible concentration gradients; Mossé, Lévy, and Adda measured the average chemical DC for a solid solution of chromium in gamma uranium with a concentration range of several atomic percent, with the diffusion taking place under a concentration gradient. The difference between these quantities can be derived from Darken's equations (13):

$$
\mathrm{D}=\mathrm{N}_{\mathrm{U}} \mathrm{D}_{\mathrm{Cr}}+\mathrm{N}_{\mathrm{Cr}} \mathrm{D}_{\mathrm{U}}
$$

and

$$
\mathrm{D}_{\mathrm{Cr}}=\mathrm{D}_{\mathrm{Cr}}^{*}\left(1+\frac{\partial \ln \gamma_{\mathrm{Cr}}}{\partial \ln N_{\mathrm{Cr}}}\right) \text {. }
$$

Since $\mathrm{N}_{\mathrm{U}} \approx 1$, and $\mathrm{D}_{\mathrm{U}} \ll \mathrm{D}_{\mathrm{Cr}}$, then $\mathrm{D} \approx \mathrm{D}_{\mathrm{Cr}}$. Here $\mathrm{N}_{\mathrm{i}}$ and $\mathrm{D}_{\mathrm{i}}$ are the mole fraction and partial $\mathrm{DC}$ of the $\mathrm{i}$ th component, $\mathrm{D}$ is the chemical $\mathrm{DC}, \mathrm{D}_{\mathrm{Cr}}^{*}$ is the DC of chromium, and $\gamma_{\mathrm{Cr}}$ the activity coefficient of chromium in a homogeneous $\mathrm{U}-\mathrm{Cr}$ solid solution. The quantities $\mathrm{D}_{\mathrm{Cr}}, \mathrm{D}_{\mathrm{Cr}}^{*}$, and $\gamma \mathrm{Cr}$ may, all be concentration dependent. We have measured $\mathrm{D}_{\mathrm{Cr}}^{*}$ for $\mathrm{N}_{\mathrm{Cr}} \approx 0$; Mossé, Lévy, and Adda have measured $\mathrm{D}_{\mathrm{Cr}}$ average over a concentration range. Thus the differences between the two sets of results could be due to the concentration dependence of $\mathrm{D}_{\mathrm{Cr}}^{*}$ or to the thermodynamic factor; the absence of data on both of these precludes further discussion.

Two other possible sources of the discrepancy are contained in the method of Mossé, Lévy, and Adda. Briefly, they prepared diffusion couples by pressure welding together pure $\mathrm{U}$ and a $\mathrm{U}-\mathrm{N}$ a/o $\mathrm{Cr}$ alloy, where $\mathrm{N}$ is a number between 2 and 6 . The couples were annealed at a temperature where the two-phase alloy was in the liquid + solid region. The value of 
$D$ was calculated from the thickness $\xi$ of the layer depleted in precipitate (Fig. 7), by means of the formulae(14):

$$
\xi=2 \gamma_{D} \sqrt{D t}
$$

and

$$
\frac{1}{\sqrt{\pi}} \frac{C_{\gamma, A}-C_{A, 0}}{C_{A}-C_{\gamma, A}}=\gamma_{D} \exp \left(\gamma_{D}^{2}\right)\left[1+\operatorname{erf} \gamma_{D}\right] \equiv \rho\left(\gamma_{D}\right),
$$

where erf $\gamma_{D}=\frac{2}{\sqrt{\pi}} \int_{0}^{\gamma_{D}} \exp \left(-t^{2}\right) d t, C_{A}$ is the concentration of chromium in the two-phase alloy, $\mathrm{C}_{\gamma, \mathrm{A}}$ is the maximum concentration of chromium in gamma uranium at the diffusion temperature, obtained from the phase diagram, and $\mathrm{C}_{\mathrm{A}, 0}$ is the concentration of chromium in the pure uranium. In practice, $\rho\left(\gamma_{D}\right)$ was determined from the phase diagram, $\gamma_{D}$ was determined from a curve of $\rho\left(\gamma_{D}\right)$ vs $\gamma_{D}$, and $D$ was determined from knowing $\xi$ as a function of $t$, knowing $\gamma_{D}$. (In a study of the diffusion of lanthanum and strontium in gamma uranium, (15) linear plots of $\xi \mathrm{D}$ vs $\sqrt{t}$ were shown, indicating that for these elements the first of the above equations was satisfied.)

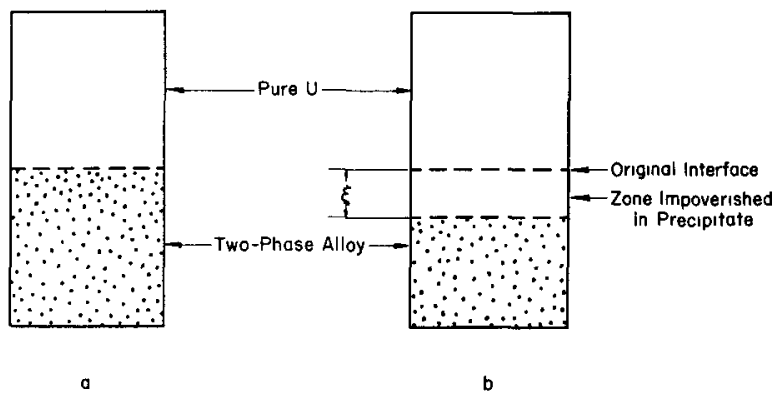

Fig. 7

Schematic Sketch of Diffusion Sample Used by Mossé, Lévy, and Adda: a. before diffusion; b. after diffusion

The accuracy of a DC determined by this method depends on the accuracy of the phase diagram; an error of 1 a/o in $\mathrm{C}_{\gamma}, \mathrm{A}$ may change $\gamma \stackrel{2}{D}$, and therefore $\mathrm{D}$, by a factor of two. Whether the data of Mosse', Le'vy, and Adda are in error due to this cause is not known; the monotonically increasing difference between the present data and theirs suggest that the slope of the solidus line used by them may have been in error.

Another possible source of error in this method is in the behavior of the liquid phase. We do not know whether the liquid phase, located largely at the grain boundaries, stayed in place, whether it flowed slowly upwards, increasing $\xi$, or whether the sample changed shape in any way. All these possibilities lead to an erroneously high DC. 
In view of uncertainties and complications such as the rmodynamic factors, concentration gradient effects, concentration-dependent DC's, uncertain phase diagrams, and the possible side effects of a liquid phase, the method of Mossé, Lévy, and Adda seems unsuitable for making measurements that yield fundamental information on the influence of atomic sizes and valences on diffusion parameters. Analogous conclusions were reached by Tomizuka and Slifkin(16) from a comparison of chemical diffusion and tracer diffusion in silver.

\section{ACKNOW LEDGMENTS}

Much of the experimental work and calculations was done by S. A. Moore. The author is obliged to Stanley Swierk for assistance with the machining, to W. G. Greenhow for programming the least-squares analyses, and to C. C. Trail for scintillating discussions of scintillation counting. 


\section{REFERENCES}

1. Mossé, M., Lévy, V., and Adda, Y., Diffusion de quelques metaux en solution diluee dans l'uranium, Compt. Rend. 250, 3171 (1960).

2. Rothman, S. J., Lloyd, L. T., Weil, R., and Harkness, A. L., Selfdiffusion in Gamma Uranium, ANL-5971 (1959); Trans. AIME 218 , 605 (1960).

3. Rothman, S. J., The Diffusion of Gold in Gamma Uranium, ANL-6127 (1960); J. Nuclear Materials 3, 77 (1961).

4. Weil, R., Rothman, S. J., and Lloyd, L. T., The Preparation of Diffusion Couples by Cathodic Sputtering, Rev. Sci. Instr. 30, 541 (1959).

5. Nuclear Data, NBS Circular 499 (Sept 1950), p. 47.

6. Mortlock, A. J., Rowe, A. H., and LeClaire, A. D., The Atomic Diffusion of Platinum in Gold, Phil. Mag. 5, 803 (1960).

7. Chiotti, P., Klepfer, H. H., and White, R. W., Lattice Parameters of Uranium from $25^{\circ} \mathrm{C}$ to $1132^{\circ} \mathrm{C}$, Trans. ASM 51, 772 (1959).

8. Armstrong, H., On Solid-state Diffusion with a Linearly Varying Temperature, Trans. AIME 212,450 (1958).

9. Lazarus, D., Effect of Screening on Solute Diffusion in Metals, Phys. Rev. 93, 973 (1954).

10. Inman, M. C., and Barr, L. W., Diffusion of Antimony in Copper Single Crystals, Acta Met. 8, 112 (1960).

11. Pound, G. M., Bitler, W. R. and Paxton, H. A., Theory of $D_{0}$ for Selfdiffusion in Solid Metals, unpublished.

12. Rothman, S. J., Gray, Jr., J., Hughes, J. P., and Harkness, A. L., Self-diffusion in Beta Uranium, ANL-6126, 1960; J. Nuclear Materials 3, $72(1961)$.

13. Darken, L. S., Diffusion, Mobility, and Their Inter relation through Free Energy in Binary Metallic Systems, Trans. AIME 175, 184 (1948).

14. Wagner, C., unpublished work, given in Jost, W., Diffusion in Solids, Liquids, and Gases, Academic Press (1952) Ch. 1, p. 68.

15. Adda, Y., Lévy, V., Hadari, Z., and Tournier, J., Methode simple pour etudier la diffusion entre deux metaux dont l'un est volatil et peu soluble dans l'autre, Mem. Sci. Rev. Met. 57, 278 (1960).

16. Tomizuka, C. T., and Slifkin, L., Diffusion of Cadmium, Indium, and Tin in Single Crystals of Silver, Phys. Rev. 96, 610 (1954). 